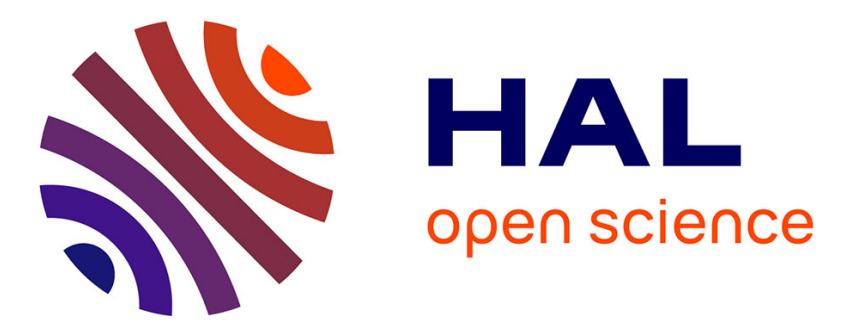

\title{
Actuator Design of Compliant Walkers via Optimal Control
}

\author{
Gabriele Buondonno, Justin Carpentier, Guilhem Saurel, Nicolas Mansard, \\ Alessandro de Luca, Jean-Paul Laumond
}

\section{- To cite this version:}

Gabriele Buondonno, Justin Carpentier, Guilhem Saurel, Nicolas Mansard, Alessandro de Luca, et al. Actuator Design of Compliant Walkers via Optimal Control. IEEE/RSJ International Conference on Intelligent Robots and Systems (IROS 2017), Sep 2017, Vancouver, Canada. 7p. hal-01483567v2

\section{HAL Id: hal-01483567 \\ https://hal.science/hal-01483567v2}

Submitted on 27 Jul 2017

HAL is a multi-disciplinary open access archive for the deposit and dissemination of scientific research documents, whether they are published or not. The documents may come from teaching and research institutions in France or abroad, or from public or private research centers.
L'archive ouverte pluridisciplinaire HAL, est destinée au dépôt et à la diffusion de documents scientifiques de niveau recherche, publiés ou non, émanant des établissements d'enseignement et de recherche français ou étrangers, des laboratoires publics ou privés.

\section{(ㄷ)(1) $\$$}

Distributed under a Creative Commons Attribution - NonCommerciall 4.0 International 


\title{
Actuator Design of Compliant Walkers via Optimal Control
}

\author{
Gabriele Buondonno ${ }^{1}$, Justin Carpentier ${ }^{2}$, Guilhem Saurel ${ }^{2}$, Nicolas Mansard ${ }^{2}$, \\ Alessandro De Luca ${ }^{1}$ and Jean-Paul Laumond ${ }^{2}$
}

\begin{abstract}
We present an optimization framework for the design and analysis of underactuated biped walkers, characterized by passive or actuated joints with rigid or non-negligible elastic actuation/transmission elements. The framework is based on optimal control, dealing with geometric constraints and various dynamic objective functions, as well as boundary conditions, which helps in selecting optimal values both for the actuation and the transmission parameters. Solutions of the formulated problems are shown for different kinds of bipedal architectures, and comparisons drawn between traditional rigid robots and compliant ones show the energy-efficiency of compliant actuators in the context of locomotion.
\end{abstract}

\section{INTRODUCTION}

Energetic efficiency in bipedal robot walking is an increasingly important line of research in robotics [1], [2]. Biology often serves as a source of inspiration to address this problem. An important characteristic of human walking patterns is that they exploit the human body's natural passive dynamics. The passive dynamics is the body dynamics when no actuation forces are present. In this case, the body is only subject to gravity, external forces, and to elastic or friction forces at joint level. The human body structure allows the main characteristics of dynamic walking to emerge naturally, thus requiring much lower energy and control frequency.

Another characteristic of the human body is the intrinsic compliance of its muscle structure. Its elastic components are capable of passively storing energy resulting from elongation, to release it at a later stage, when it is more useful. This allows the body to significantly increase efficiency in legged locomotion [3].

The human passive walking dynamics is trying to be imitated by passive walkers, of which many different prototypes exist today [4], [5]. In principle, this specific type of biped robots is able to maintain stability without any action from the motors, which can be entirely absent, and is able to walk along an inclined plane pushed by gravity only. Clearly, a real robot, in order to serve any practical purpose, cannot be entirely passive, but will need to possess some kind of actuation; thus, the guiding principle will be

\footnotetext{
${ }^{1}$ Dipartimento di Ingegneria Informatica, Automatica e Gestionale, Sapienza Università di Roma, Via Ariosto 25, 00185 Rome, Italy \{buondonno,deluca\}@diag.uniroma1.it

${ }^{2}$ LAAS-CNRS, Université de Toulouse, CNRS, Toulouse, France \{jcarpent,gsaurel,nmansard,jpl\}@ @laas.fr

This work is supported by the European Research Council (ERC) through the Actanthrope project (https://actanthrope.laas.fr/) (ERC grant agreement 340050), the French National Research Agency through the Loco3D project (Project ID: ANR-16-CE33-0003), and the European Commission through the $\mathrm{H} 2020$ project 645097 COMANOID.
}

that of optimizing the design so that the actuator effort during nominal walking be minimal.

The idea of introducing compliance was a direct inspiration for Series Elastic Actuators (SEA) [6], as found in [7]. This is a new generation of actuators and transmission elements characterized by non-negligible elasticity of the transmission elements between the motors and the respective links. Their application is not only limited to manipulator arms, but it has been successfully applied to complex bipedal structures such as the TORO robot [8], developed by the DLR. By contrast, in Parallel Elastic Actuators [9] the motors directly drive the respective links through rigid transmission elements, but in addition the link is also connected to a fixed point through a spring. Recently, the tendency is to go even further and shift to Variable Impedance Actuators [10], characterized by controllable stiffness and damping parameters; an example is the HASY bimanual hand-arm system [11], [12], still by the DLR.

On the control side, it has been noticed how intrinsic compliance can considerably simplify the task of interacting with the environment, as it allows the robot configuration to naturally adapt to unmodeled circumstances [13]. The possibility to exploit the energy storage provided by the elastic elements was demonstrated in [14]. Further studies showed how elastic joints can produce very explosive motions, if conveniently controlled [15]. Clearly, intrinsic compliance is not an advantage per se, but it needs to be adequately exploited by control [16]. Thus, any study about elastic-joint robots cannot leave control out of consideration. On the other hand, the way elastic elements can influence passive walking has not been deeply investigated yet.

Traditionally, the design of passive walkers is a long and elaborated process, essentially left to the ability and experience of the designer. Concerning the sizing of the actuators, important work was done in the VIACTORS project [17], [18]. The problem becomes even more sensitive if elastic elements are inserted into the dynamics, the stability analysis of the systems being highly complex. Because of this, the capabilities of VIA-equipped robots are often limited to very simple settings, making them unsuited for more general environments. Therefore, there is a need to develop a software capable to automatize and speed up at least part of the design process, in particular the study of the different types of structures and actuators, the selection of the dynamic parameters of the robot itself, and the analysis of the relationship between these two choices.

This is the basic idea of co-design. A first step in this direction was taken in [19]. In that work, the 
authors introduced a framework for simultaneous design and control of passivity-based walkers, and they applied it to different kinds of kinematic structures to draw quantitative comparisons between them. The framework presented there is based on mathematical optimization: the design problem is formulated as an optimal control problem (OCP), which is potentially capable to optimize the state trajectory, the input trajectory and the robot parameters together in order to minimize a given cost function, subject to various path constraints. In this work, we optimize the robot trajectory and some related parameters, while the dynamical parameters of the robot itself are left untouched.

Our different contributions include

- The presentation of an optimization framework allowing to design and simulate robots equipped with different kinds of elastic actuators. This is based on the experience of [19].

- The framework is shown to be capable of simulating whole-body humanoids equipped with elastic actuators.

- Different performance criteria are implemented. Simulation results include cost of transport (CoT) and torque minimization.

Among previous works featuring compliant humanoids simulation, we note [20] and [21]. The paper is organized as follows. In Sec. II, we present the dynamics of general biped robots, with the different kinds of actuators. In Sec. III, we illustrate the framework. In Sec. IV, we show the output of some simulations, both on a simple model and on a more complex whole-body model, for different cost functions, employing traditional rigid actuation, SEAs, and parallel elastic actuators, drawing comparisons between the different cases. Final considerations are conducted in Sec. V.

\section{DYNAMICS}

In this paper, we assume a punctual rigid contact between the robot stance foot and the ground. Under this hypothesis, the continuous dynamics of a robotic walker is adequately modeled as that of a fixed-base kinematic tree, provided non-slippage constraints are respected. In the model, the robot stance ankle will be replaced by a single unactuated joint directly connected to the ground; this will preserve the inherent underactuation of a biped robot. If only the sagittal dynamics is concerned, this will be a revolute joint whose axis is perpendicular to the sagittal plane; if the lateral dynamics is also taken into account, it will be a 3-DoF (3 Degrees of Freedom) spherical joint. In the following, we are going to describe the dynamical equations of such systems. The impact dynamics can also be considered during phase transitions, as shown in [19], but we will leave it out for the purpose of this paper.

\section{A. Rigid robots model}

Consider first a possibly underactuated robot consisting of a kinematic tree of $n$ rigid links connected through $n$ rigid joints, equipped with $m \leq n$ motors, maximum one per link. The classical Lagrangian model of such a system is given by

$$
\boldsymbol{M}(\boldsymbol{q}) \ddot{\boldsymbol{q}}+\boldsymbol{c}(\boldsymbol{q}, \dot{\boldsymbol{q}})+\boldsymbol{g}(\boldsymbol{q})=\boldsymbol{S}^{T} \boldsymbol{\tau}
$$

where $\boldsymbol{q} \in \mathbb{R}^{n}$ is the vector of link positions, $\boldsymbol{\tau} \in \mathbb{R}^{m}$ is the vector of motor torques (with $m \leq n$ ), $\boldsymbol{S} \in \mathbb{R}^{m \times n}$ is a selection matrix encoding the underactuation, $\boldsymbol{M}(\boldsymbol{q})$ is the joint space inertia matrix, $\boldsymbol{c}(\boldsymbol{q}, \dot{\boldsymbol{q}})$ is the vector of centrifugal and Coriolis forces, and $\boldsymbol{g}(\boldsymbol{q})$ is the vector of gravity forces. The dynamic state of the robot is given by the link variables and their time derivatives, namely:

$$
\boldsymbol{x}=\left(\begin{array}{ll}
\boldsymbol{q}^{T} & \dot{\boldsymbol{q}}^{T}
\end{array}\right)^{T} \in \mathbb{R}^{N}, \quad N=2 n .
$$

\section{B. Series elastic actuators model}

In the case of SEAs, the position of the motors is not directly linked to that of the links, and must therefore be represented separately. In the following we will assume linear spring elasticity, which is reasonable if the spring deflections are small. Moreover, for simplicity we will employ the so-called reduced model of elastic-joint robots, which is obtained by neglecting all kinetic energy contributions due to the inertial couplings between the motors and the links; this working hypothesis, obtained by Spong [22], has been put to test repeated times and has proven to be very effective in practice. Under these assumptions, the model changes as the following

$$
\begin{aligned}
& \boldsymbol{M}(\boldsymbol{q}) \ddot{\boldsymbol{q}}+\boldsymbol{c}(\boldsymbol{q}, \dot{\boldsymbol{q}})+\boldsymbol{g}(\boldsymbol{q})=\boldsymbol{S}^{T} \boldsymbol{K}(\boldsymbol{\theta}-\boldsymbol{S q}) \\
& \boldsymbol{J \boldsymbol { \theta }}+\boldsymbol{K}(\boldsymbol{\theta}-\boldsymbol{S q})=\boldsymbol{\tau} \\
& \boldsymbol{x}=\left(\boldsymbol{q}^{T} \dot{\boldsymbol{q}}^{T} \boldsymbol{\theta}^{T} \dot{\boldsymbol{\theta}}^{T}\right)^{T} \in \mathbb{R}^{N}, \quad N=2(n+m)
\end{aligned}
$$

where $\boldsymbol{\theta} \in \mathbb{R}^{m}$ is the vector of motor positions, after the reduction gears, i.e. divided by the reduction ratio. Equations (3a) and (3b) are referred to as link equation and motor equation, respectively. Here, $\boldsymbol{K} \in \mathbb{R}^{m \times m}$ and $\boldsymbol{J} \in \mathbb{R}^{m \times m}$ are positive-definite diagonal matrices containing, respectively, the stiffness of the transmission elements and the so-called drive inertia moments, i.e. the inertias of the motor rotors around their own spinning axes multiplied by the squared reduction ratio. Also, the vector of joint deflections is given as $\boldsymbol{\phi}=\boldsymbol{\theta}-\boldsymbol{S} \boldsymbol{q}$.

\section{Parallel elastic actuators model}

Finally, for parallel elastic actuators, the Lagrangian model is

$$
\begin{aligned}
& \boldsymbol{M}(\boldsymbol{q}) \ddot{\boldsymbol{q}}+\boldsymbol{c}(\boldsymbol{q}, \dot{\boldsymbol{q}})+\boldsymbol{g}(\boldsymbol{q})=\boldsymbol{S}^{T}(\boldsymbol{\tau}+\boldsymbol{K}(\overline{\boldsymbol{q}}-\boldsymbol{q})) \\
& \boldsymbol{x}=\left(\boldsymbol{q}^{T} \dot{\boldsymbol{q}}^{T}\right)^{T} \in \mathbb{R}^{N}, \quad N=2 n
\end{aligned}
$$

where $\bar{q}$ is the constant equilibrium point of the springs, and it can be seen that $\boldsymbol{x}$ is as in (2). In order not to compromise the mobility of the robot, the joint stiffness will be typically very low.

\section{FRAMEWORK}

In this section, we setup the co-design problem using the tools of optimal control. We will design the control problem over the duration of a step.

In general, the goal of the optimal control problem is to minimize the integral of a cost function $c(\boldsymbol{x}, \boldsymbol{\tau})$ over the whole step duration, depending on the state trajectory, the control trajectory, and some parameters $\boldsymbol{p}$, subject to some 


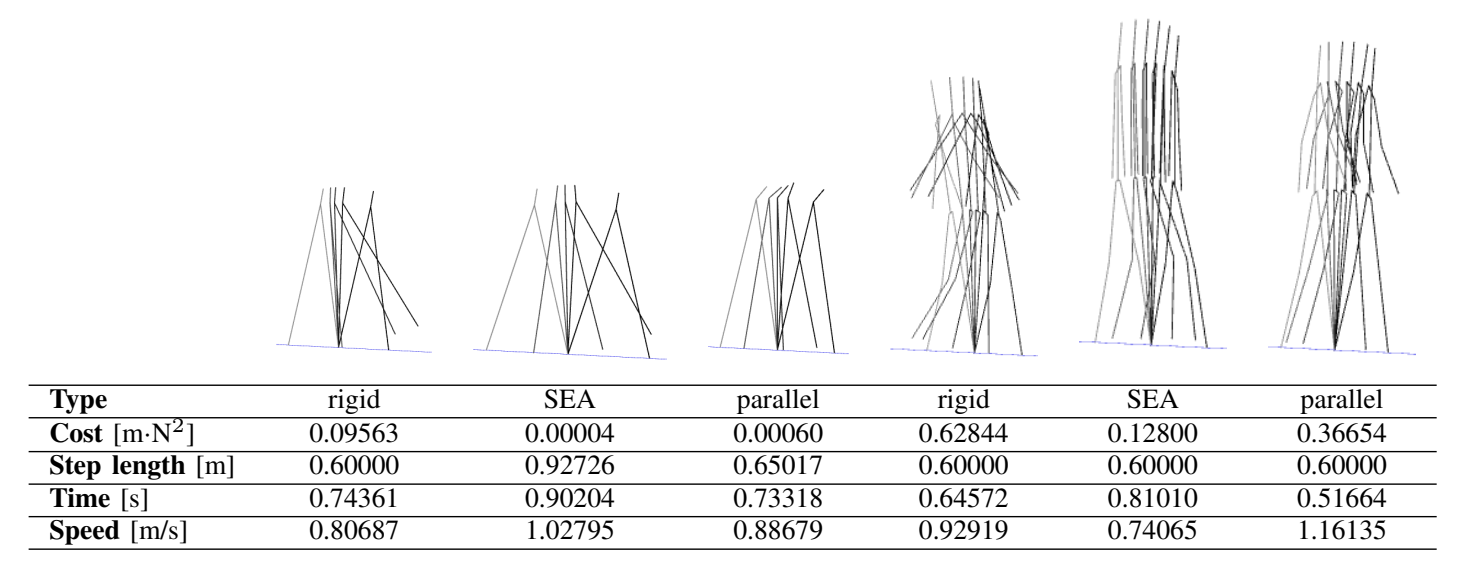

TABLE I: Output of the simulations employing the squared norm of the torque as objective function.

constraints. These constraints are divided into decoupled constraints $f_{d}$, which have to be valid at each trajectory point, and coupled constraints $f_{c}$, which relate the initial and final trajectory point to ensure periodicity of the solution and other high-level trajectory properties.

\section{A. Rigid robots}

For rigid robots, the problem is set up as

$$
\begin{gathered}
\min _{\underline{\boldsymbol{x}}, \underline{\boldsymbol{\tau}}, \boldsymbol{p}} \int_{0}^{T} c(\boldsymbol{x}, \boldsymbol{\tau}, \boldsymbol{p}) \mathrm{d} t \\
\text { s.t } \\
\ddot{\boldsymbol{q}}=\boldsymbol{M}^{-1}(\boldsymbol{q})\left(\boldsymbol{S}^{T} \boldsymbol{\tau}-\boldsymbol{c}(\boldsymbol{q}, \dot{\boldsymbol{q}})-\boldsymbol{g}(\boldsymbol{q})\right) \\
\boldsymbol{f}_{d}(\boldsymbol{x}, \boldsymbol{\tau}) \geq 0 \\
\boldsymbol{f}_{c}(\boldsymbol{x}(0), \boldsymbol{\tau}(0), \boldsymbol{x}(T), \boldsymbol{\tau}(T), \boldsymbol{p})=0
\end{gathered}
$$

with $\underline{x}$ and $\underline{\tau}$ denoting the state and control trajectories respectively. The parameters $\boldsymbol{p}$ mostly include trajectory and environment properties, e.g. the step length and the inclination of the slope along which the robot walks. The decoupled constraints $f_{d}$ include bounds on all variables, while the coupled constraints $f_{c}$ enforce, other than periodicity constraints, the step length.

The choice of the "best" cost function is still an open problem in robotics. In this paper, we explore two different alternatives.

The first possibility is the so-called cost of transport (CoT). This is equal to the ratio between the energy consumed by the system and the weight multiplied by the travel distance. The consumed energy is equal to the difference in potential energy plus the integral of the power input. If the slope is fixed, this is equivalent to minimizing

$$
c(\boldsymbol{x}, \boldsymbol{\tau})=c_{\mathrm{CoT}}=\frac{|\boldsymbol{\tau}|^{T} \boldsymbol{S}|\dot{\boldsymbol{q}}|}{L}
$$

where $L$ is the step length, and $|\cdot|$ is the component-wise absolute value. Here, we are assuming energy cannot be recovered, which is why we included the absolute values. This may resemble human dynamics more closely. Notice that, on some modern hardware, such as the Cheetah robot [23], some energy can be recovered from braking.
However, this is always at a huge loss, most of the energy going to be lost anyways. This was the cost function of choice in [19], as well as in classical works such as [24].

Another possibility is the squared norm of the torque, normalized by the step length:

$$
c(\boldsymbol{x}, \boldsymbol{\tau})=c_{\boldsymbol{\tau}}=\frac{\|\boldsymbol{\tau}\|^{2}}{L} .
$$

This choice of function is useful to keep the torques exerted by the motors limited, and may in general lead to better-conditioned problems. It has already been observed that penalizing the squared norm of the torque variable instead of the mechanical work produces behaviors which tend to appear more natural [25].

\section{B. Series elastic actuators}

The optimal control problem for SEAs is set up similarly, taking into account the different dynamics (3). Care should be taken in ensuring the periodicity of $\boldsymbol{\theta}$ and $\dot{\boldsymbol{\theta}}$ too. Additionally, we optimize the parameters for $\boldsymbol{K}$ and $\boldsymbol{J}$. It should be noticed that we will want the parameters of some joints to coincide with each other. For instance, the joints at both hips should be characterized by the same stiffness and drive inertia moment, and the same can be said for possible knees, shoulders and elbows. Rather than enforcing this as a constraint, we will use a single parameter for the stiffness of each couple of symmetric joints, and one for the drive inertia moment, thus reducing the dimensionality of the problem and enhancing precision.

The mathematical expression of the CoT is slightly changed, becoming

$$
c_{\mathrm{CoT}}=\frac{|\boldsymbol{\tau}|{ }^{T}|\dot{\boldsymbol{\theta}}|}{L} .
$$

Notice that, since it is assumed that the final pose is exactly specular to the initial one, no contribution to the cost function is assumed from the potential elastic energy. Also, it should be ensured that the spring deflections do not exceed certain limits:

$$
|\boldsymbol{\theta}-\boldsymbol{S} \boldsymbol{q}|<\boldsymbol{\phi}_{\max } .
$$




\begin{tabular}{c|cc|cc}
\hline & \multicolumn{2}{|c|}{ Series elastic actuators } & \multicolumn{2}{c}{ Parallel elastic actuators } \\
\hline & $\boldsymbol{K}[\mathrm{Nm} / \mathrm{rad}]$ & $\boldsymbol{J}\left[\mathrm{kg} \mathrm{m}^{2}\right]$ & $\boldsymbol{K}[\mathrm{Nm} / \mathrm{rad}]$ & $\overline{\boldsymbol{q}}[\mathrm{rad}]$ \\
\hline compass & 1254.678 & 0.724 & 11.128 & 0.484 \\
\hline knees & 1642.951 & 0.500 & 51.829 & -0.032 \\
\hline hips & 1564.161 & 0.434 & 17.847 & -0.121 \\
\hline neck & 1434.195 & 0.566 & 61.210 & 0.040 \\
\hline shoulders & 1388.581 & 0.573 & 0.000 & 0.021 \\
\hline elbows & 1480.827 & 0.426 & 30.969 & -0.189 \\
\hline
\end{tabular}

TABLE II: Dynamic parameters of the compliant actuators, optimized w.r.t the squared torque cost function. On the first line, the values for the compass walker are shown, equal or symmetrical for both hips. In the rest of the table, the values for the joints of the complete walker are shown.

\section{Parallel elastic actuators}

For parallel elastic actuators, we optimize $\boldsymbol{K}$ and $\overline{\boldsymbol{q}}$, still ensuring symmetry of the parameters. In order not to constrain the mobility of the robot, we do not put bounds on the deflections, relying on the low stiffness. The CoT is still expressed as in (6).

\section{Implementation}

In order to solve the optimal control problem, we employ MUSCOD-II [26], a general-purpose solver based on multiple shooting, developed by the Optimization and Simulation group at the University of Heidelberg. Multiple shooting is a powerful optimization technique which divides the integration interval into a fixed number of multiple-shooting intervals, separated by nodes. Each subinterval is additionally discretized and integrated independently, ensuring continuity at the nodes. The convergence properties of multiple shooting and its faithful representation of the system dynamics make it preferable to other methods commonly used, such as single shooting and collocation; see [19] for a deeper discussion.

To compute the evolution of the system, we rely on the $\mathrm{C}++$ dynamics library Pinocchio [27], which is an efficient open source implementation of the dynamics of general polyarticulated systems, developed at LAAS-CNRS.

\section{Results}

In the following, we demonstrate the effectiveness of our proposed framework over two different robot structures. The first one is a simple planar compass model, with only two joints, one per hip. For this model, 9 multiple-shooting nodes where employed. The second one corresponds to a full humanoid in 3D, fitted with knees, hips, neck, shoulders and elbows, with a total of 12 DoFs (including 3 at the stance ankle). Here, we used 10 multiple-shooting nodes. For the two structures, we test rigid joints, series-elastic actuators and parallel-elastic actuators. We optimize both the step length and the time duration, as well as the intrinsic parameters of the actuators in the case of compliantly-actuated models. The step length has a lower bound of $0.6 \mathrm{~m}$. Concerning the inertial parameters, they follow a standard human distribution.

The simulations are divided into two series: in the first one, we employ the squared norm cost function (7), while in
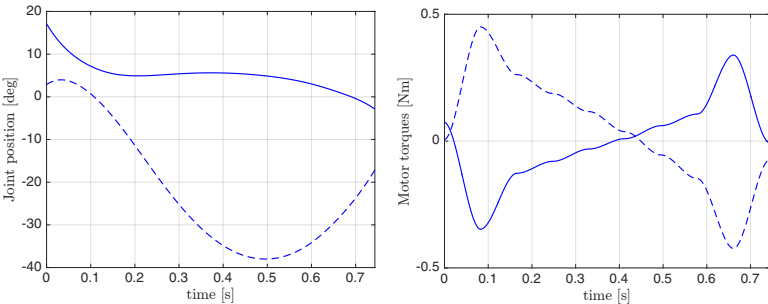

(a) Joint position and torque in the case of rigid actuation
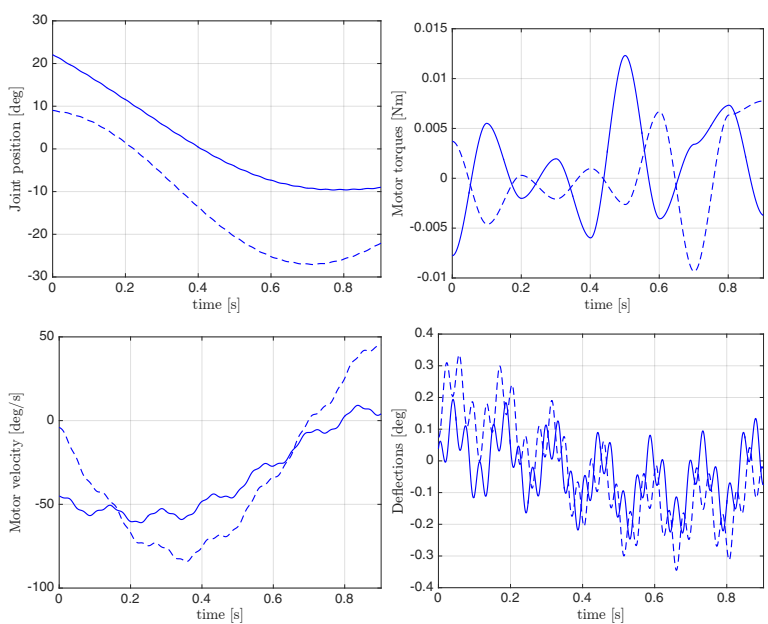

(b) Joint position, motor velocity, joint torque and spring deflection in the case of series-elastic actuation
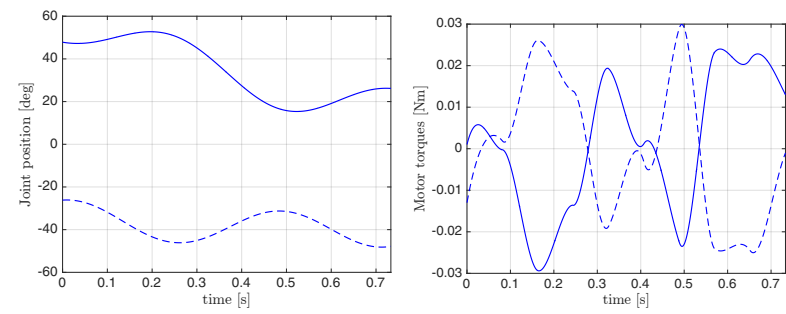

(c) Joint position and torque in the case of parallal-elastic actuation

Fig. 1: Plots of the joint positions and torques trajectories for the simple walker with the squared norm of the torque as objective function. Legend: solid=stance hip, dashed=free hip.

the second one we employ the CoT (6) or (8) depending on the type of actuators. For all scenarios, we use 10 mutiple-shooting nodes; the maximum computation time to solve the OCP is around $1 \mathrm{~min}$ for the more complex walker. We refer the reader to the accompanying video to fully appreciate the resulting gaits.

\section{A. Squared torque cost function}

In this subsection, we study the influence of the squared torque cost function for the two proposed models with the three types of actuators. The output of the optimizer is displayed in Tab. I and the optimized parameters for the compliant models are shown in Tab. II.

1) Compass walker: From Fig. 1, it appears that the torques exerted by the motors in the context of elastic models are much smaller than the ones exerted in the rigid model. Consequently, the values of the cost function for elastic 

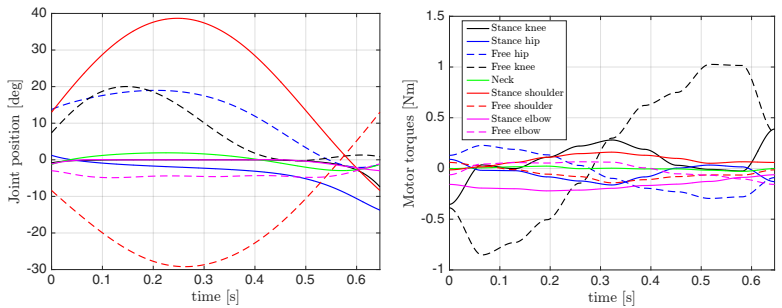

(a) Joint position and torque in the case of rigid actuation
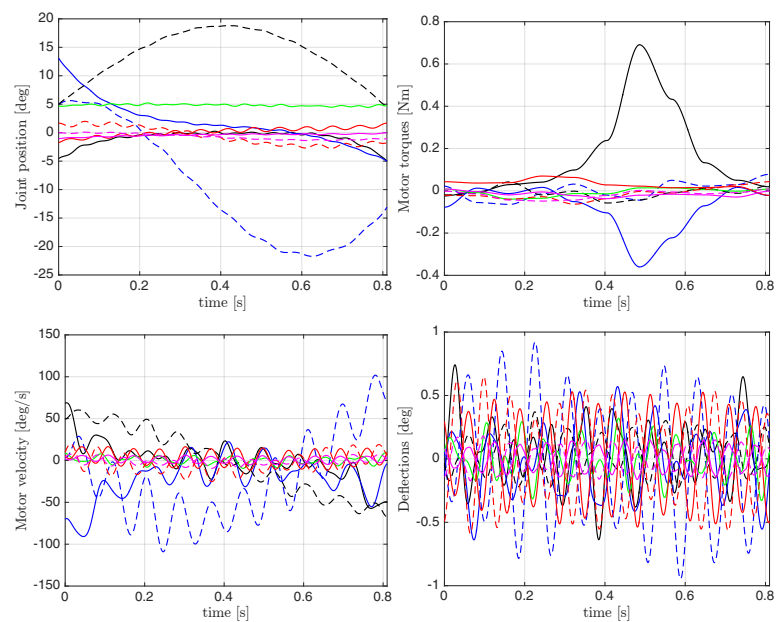

(b) Joint position, motor velocity, joint torque and spring deflection in the case of series-elastic actuation
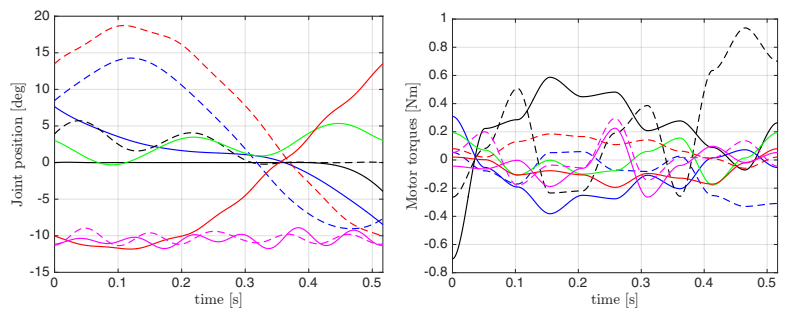

(c) Joint position and torque in the case of parallel actuation

Fig. 2: Plots of the joint positions and torque trajectories for the full walker with the squared norm of the torque as objective function. Legend: solid $/$ dashed $=$ stance $/$ swing, black $=$ knees, blue $=$ hips, green $=$ neck, red $=$ shoulders, magenta $=$ elbows .

models are quite similar and much smaller than those of the rigid example (see Tab. I, first row). For all cases, the joint trajectory is reasonably smooth. In the context of serial actuation, the joint deflection is kept within a small range, with high frequency oscillations appearing in the joint trajectory. On the other hand, the motor velocity is subject to strong oscillations. This is not surprising, since the optimal solution need not be smooth, and oscillatory behavior is typical of SEAs.

2) Full walker: We now focus on the rigidly-actuated complete walker. From Tab. I, we see that the motion appears to be very natural, also exploiting the arms to obtain more thrust, while the elbows are barely moving (see the accompanying video). From Fig. 2a, we see that the exerted torques are very low, the largest one being at the free knee, reaching a peak of about $\pm 1[\mathrm{Nm}]$. The
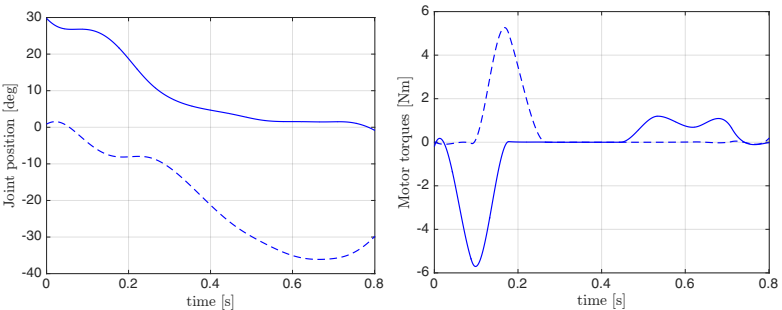

(a) Joint position and torque in the case of rigid actuation
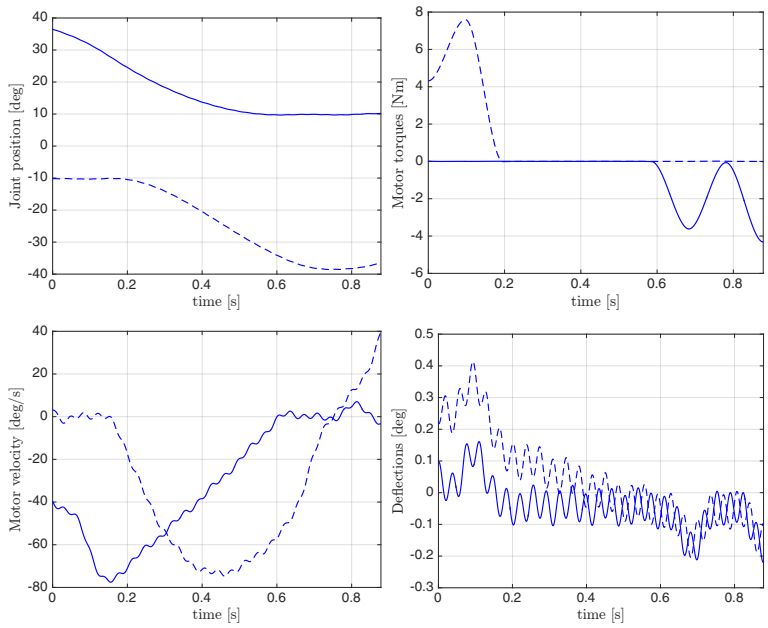

(b) Joint position, motor velocity, joint torque and spring deflection in the case of series-elastic actuation
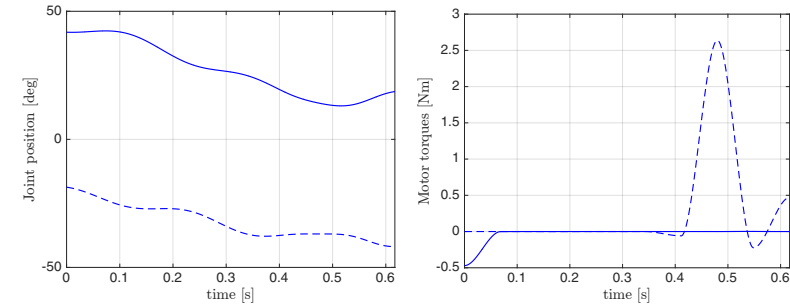

(c) Joint position and torque in the case of parallel-elastic actuation

Fig. 3: Plots of the joint positions and torques trajectories for the simple walker with the cost of transport as objective function.

nominal performance again improves using SEAs. From Fig. $2 b$, we notice some visible, small oscillations in the joint configuration, particularly in the shoulders and the neck. Also, notice that the joint deflections are below one degree. Further, we can see (also from Tab. I) that the swing motion of the arms is reduced. The performance of the parallel-elastic actuated robot is also good. Again, we can notice the role of the arms during motion. It is worth to notice that in Tab. II the spring stiffness value reaches zero for the shoulder parallel-elastic actuator. It is clear that this means that in this case the parallel elastic actuator has become a rigid one.

\section{B. Cost of transport objective function}

In this subsection, we study the influence of the cost of transport as objective function on the various case studies. Tables III and IV, as well as Figs. 3 and 4 show the output of this second series of simulations. 


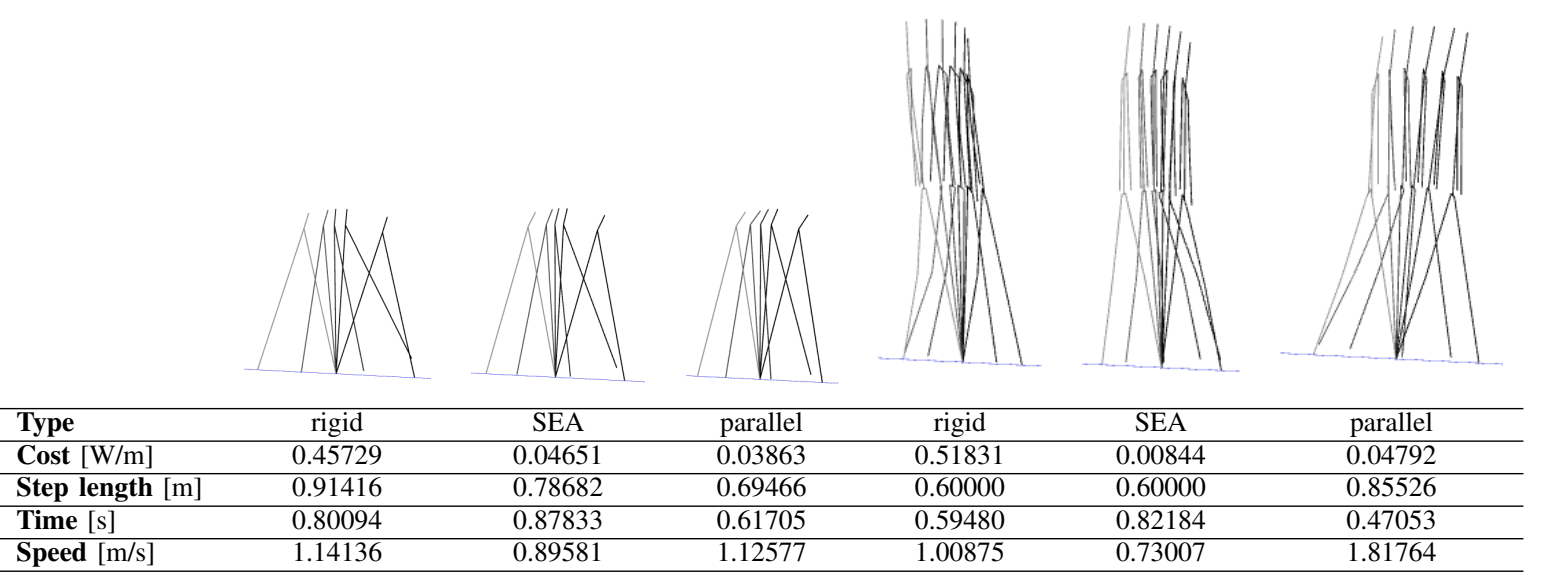

TABLE III: Output of the simulations employing the cost of transport as objective function.

\begin{tabular}{c|cc|cc}
\hline & \multicolumn{2}{|c|}{ Series elastic actuators } & \multicolumn{2}{c}{ Parallel elastic actuators } \\
\hline & $\boldsymbol{K}[\mathrm{Nm} / \mathrm{rad}]$ & $\boldsymbol{J}\left[\mathrm{kg} \mathrm{m}^{2}\right]$ & $\boldsymbol{K}[\mathrm{Nm} / \mathrm{rad}]$ & $\overline{\boldsymbol{q}}[\mathrm{rad}]$ \\
\hline compass & 1231.374 & 0.308 & 37.648 & 0.485 \\
\hline knees & 1588.078 & 0.758 & 129.195 & -0.017 \\
\hline hips & 1378.095 & 0.280 & 44.888 & -0.069 \\
\hline neck & 1415.037 & 0.479 & 60.235 & 0.085 \\
\hline shoulders & 1485.496 & 0.638 & 131.771 & -0.055 \\
\hline elbows & 1341.142 & 0.479 & 37.859 & -0.011
\end{tabular}

TABLE IV: Dynamic parameters of the compliant actuators, optimized w.r.t the CoT cost function.

1) Compass walker: For the compass walker with CoT as objective function, higher frequency oscillations appear on the SEAs. In the meantime, all the torque trajectories have similar amplitude. At this stage, it is worth to mention that during a non-negligible amount of time the torque trajectories are identically equal to zero for both hips, although the peak values are higher. This means that, during this period, the OCP solver manages to find out a strategy to exploit the walker's intrinsic dynamics only, which was not the case with the previous cost function.

2) Full walker: The same patterns as is the previous series apply here, with some exceptions. In general, we see that the arms are not moving, but lie rigidly next to the body; the parallel-elastic actuated walker is holding them slightly to its front. Many joints are left almost unactuated (practically all of them except the knees and one elbow for the SEA case). It should be noted that minimizing the CoT required in general much more computation time than minimizing the squared torque.

\section{Serial vs. parallel actuations}

For both cost functions, it appears SEAs produce high oscillations of the motor shafts. In other words, with such kind of actuation both the electronics and the motor are highly stressed by the spring element and must be designed accordingly. In addition, comparing stiffness values in Tabs. II and IV, it appears that SEAs need higher stiffness (bigger by one order of magnitude). Then springs occupy a larger volume in the robot in the serial case.

For all simulations, it is worth to notice that parallel elastic actuators unlike SEAs tend to make the mean motor torque value close to zero. In other words, with serial elastic actuators, motors have to support a greater burden. This also means that optimal control allows to correctly exploit the spring as storage entity in the context of parallel actuators and minimally stress the motors.

\section{CONCLUSION AND Future Works}

In this work, we have presented a framework capable to design and analyze the actuation system of complex robots. We have tested it over different robot structures, with various cost functions, showing that in general our framework is able to properly exploit the elastic potential energy along the complete trajectory.

The framework can be extended in different ways. In the first place, the selection of the type of actuator should be automatic for each joint; the most straightforward implementation of this concept would be setting up a mixed-integer optimal control problem (MIOCP) which will choose the optimal actuator combination minimizing the objective [28], [29]. Actuators individually combining serial and parallel elastic connections could also be interesting. The increase of certain parameters such as the stiffness should also correspond to a greater mass. Different tasks such as jumping or running can be studied. Another extension could be the implementation of a strategy to avoid local minima. A Montecarlo method on the initial values seems the most promising approach. A stochastic solution may also be employed to achieve robustness.

The most open and promising aspect consists in a deep study of not only the mechanics of the robot, but also its electromechanics. This might be required in order to account for low-level limitations in, e.g., current, tension and tracking controllers. Also, the design of a cost function that faithfully reflects the actual energy consumption of the robot might involve modelling the electromechanics, for instance to include the contribution of motors and regenerative system as in Cheetah robot [23]. Depending on how much the energy consumption model of the robot is similar to that of human beings, the resulting gaits may or may not be human-like. One might wonder if it is even desirable, then, that a robot exclusively pursue energy efficiency without regard for the biological inspirations. Most likely, the answer will depend on the application. 

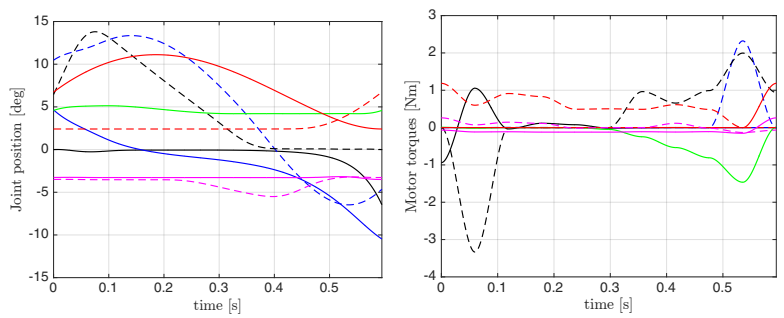

(a) Joint position and torque in the case of rigid actuation
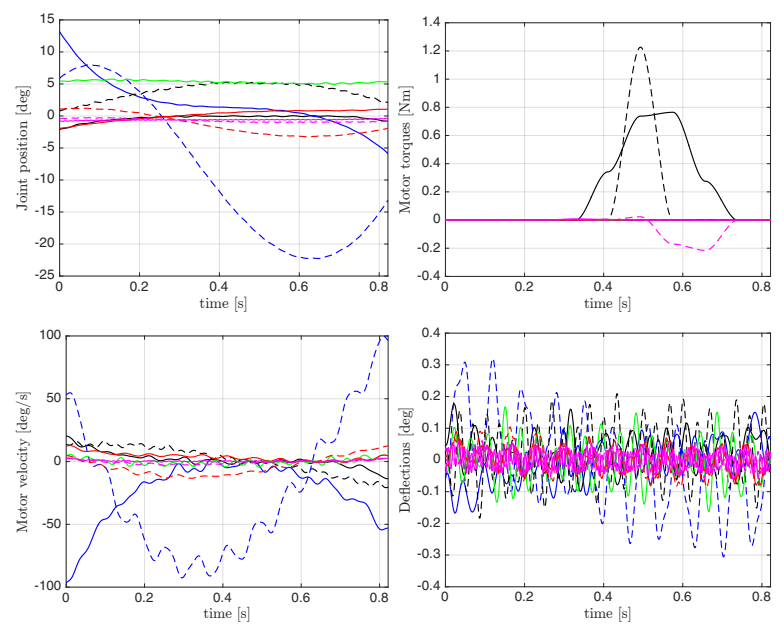

(b) Joint position, motor velocity, joint torque and spring deflection in the case of series-elastic actuation
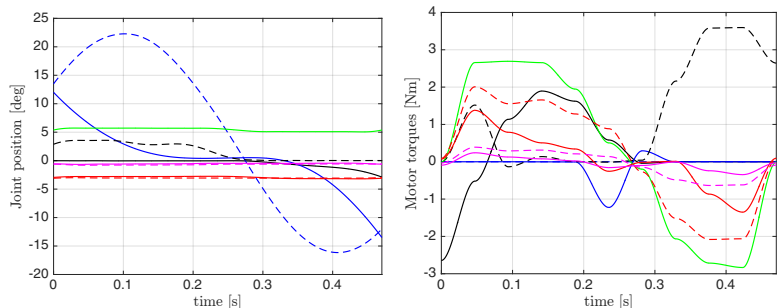

(c) Joint position and torque in the case of parallel actuation

Fig. 4: Plots of the joint positions and torques trajectories for the full walker with the cost of transport as objective function.

\section{ACKNOWLEDGEMENT}

We warmly thank the Simulation and Optimization group at the Interdisciplinary Center for Scientific Computing (IWR) of Heidelberg University in Germany for providing the optimal control framework MUSCOD-II.

\section{REFERENCES}

[1] S. Collins, A. Ruina, R. Tedrake, and M. Wisse, "Efficient bipedal robots based on passive-dynamic walkers," Science, vol. 307, no. 5712, pp. 1082-1085, 2005.

[2] P. A. Bhounsule et al., "Low-bandwidth reflex-based control for lower power walking: $65 \mathrm{~km}$ on a single battery charge," Int. J. of Robotics Research, vol. 33, no. 10, pp. 1305-1321, 2014.

[3] R. M. Alexander, Elastic Mechanisms in Animal Movement. Cambridge University Press, 1988.

[4] M. Wisse and R. Q. Van der Linde, Delft pneumatic bipeds. Springer Science \& Business Media, 2007, vol. 34.

[5] Y. Ikemata, A. Sano, and H. Fujimoto, "A physical principle of gait generation and its stabilization derived from mechanism of fixed point," in Proc. IEEE Int. Conf. on Robotics and Automation, 2006, pp. 836-841.
[6] G. Pratt and M. Williamson, "Series elastic actuators," in Proc. IEEE/RSJ Int. Conf. on Intelligent Robots and Systems, 1995, pp. 399-406.

[7] T. D. Tuttle and W. P. Seering, "A nonlinear model of a harmonic drive gear transmission," IEEE Trans. on Robotics and Automation, vol. 12, no. 3, pp. 368-374, 1996.

[8] J. Englsberger et al., "Overview of the torque-controlled humanoid robot TORO," in Proc. IEEE-RAS Int. Conf. on Humanoid Robots, 2014, pp. 916-923.

[9] M. Grimmer, M. Eslamy, S. Gliech, and A. Seyfarth, "A comparison of parallel- and series elastic elements in an actuator for mimicking human ankle joint in walking and running," in Proc. IEEE Int. Conf. on Robotics and Automation, 2012, pp. 2463-2470.

[10] B. Vanderborght et al., "Variable impedance actuators: A review," Robotics and Autonomous Systems, vol. 61, no. 12, pp. 1601-1614, 2013.

[11] [Online]. Available: http://www.dlr.de/rmc/rm/en/desktopdefault.aspx/ tabid-11666/\#gallery/28234

[12] Grebenstein et al., "The DLR Hand Arm System," in Proc. IEEE Int. Conf. on Robotics and Automation, 2011, pp. 3175-3182.

[13] M. Catalano, G. Grioli, E. Farnioli, A. Serio, C. Piazza, and A. Bicchi, "Adaptive synergies for the design and control of the Pisa/IIT SoftHand," Int. J. of Robotics Research, vol. 33, no. 5, pp. 768-782, 2014.

[14] S. Haddadin, K. Krieger, M. Kunze, and A. Albu-Schäffer, "Exploiting potential energy storage for cyclic manipulation: An analysis for elastic dribbling with an anthropomorphic robot," in Proc. IEEE/RSJ Int. Conf. on Intelligent Robots and Systems, 2011, pp. 1789-1796.

[15] M. C. Özparpucu and S. Haddadin, "Optimal control for maximizing link velocity of visco-elastic joints," in Proc. IEEE/RSJ Int. Conf. on Intelligent Robots and Systems, 2013, pp. 3035-3042.

[16] D. J. Braun et al., "Robots driven by compliant actuators: Optimal control under actuation constraints," IEEE Trans. on Robotics, vol. 29, no. 5 , pp. 1085-1101, 2013.

[17] [Online]. Available: http://www.viactors.org/

[18] G. Grioli et al., "Variable stiffness actuators: The user's point of view," Int. J. of Robotics Research, vol. 34, no. 6, pp. 727-743, 2015.

[19] G. Saurel, J. Carpentier, N. Mansard, and J.-P. Laumond, "A simulation framework for simultaneous design and control of passivity based walkers," in Proc. IEEE Int. Conf. on Simulation, Modeling, and Programming for Autonomous Robots, 2016, pp. 105-110.

[20] H. Dallali, "Modelling and dynamic stabilisation of a compliant humanoid robot, CoMan," PhD thesis, University of Manchester, 2011.

[21] E. Spyrakos-Papastavridis, D. G. Caldwell, and N. G. Tsagarakis, "Balance and impedance optimization control for compliant humanoid stepping," in Proc. IEEE/RSJ Int. Conf. on Intelligent Robots and Systems, 2016, pp. 1349-1355.

[22] M. Spong, "Modeling and control of elastic joint robots," ASME J. of Dynamic Systems, Measurement, and Control, vol. 109, no. 4, pp. 310-319, 1987.

[23] S. Seok et al., "Design principles for highly efficient quadrupeds and implementation on the MIT Cheetah robot," in Proc. IEEE Int. Conf. on Robotics and Automation, 2013, pp. 3307-3312.

[24] E. Schuitema, D. G. Hobbelen, P. P. Jonker, M. Wisse, and J. D. Karssen, "Using a controller based on reinforcement learning for a passive dynamic walking robot," in Proc. IEEE-RAS Int. Conf. on Humanoid Robots, 2005, pp. 232-237.

[25] G. Schultz and K. Mombaur, "Modeling and optimal control of human-like running," IEEE/ASME Trans. on Mechatronics, vol. 15, no. 5, pp. 783-792, 2010.

[26] D. B. Leineweber, I. Bauer, H. G. Bock, and J. P. Schlöder, "An efficient multiple shooting based reduced SQP strategy for large-scale dynamic process optimization. Part 1: Theoretical aspects," Computers \& Chemical Engineering, vol. 27, no. 2, pp. 157-166, 2003.

[27] J. Carpentier, F. Valenza, N. Mansard et al. (2015) Pinocchio: Fas forward and inverse dynamics for poly-articulated systems. [Online]. Available: https://stack-of-tasks.github.io/pinocchio

[28] S. Sager, Numerical methods for mixed-integer optimal control problems. Der Andere Verlag, 2005.

[29] C. Kirches, "Mixed-integer optimal control," in Fast Numerical Methods for Mixed-Integer Nonlinear Model-Predictive Control. Springer, 2011, pp. 31-60. 